\title{
Research Article \\ Wireless Pressure Sensor Assisted Orthopedic Nursing Effectiveness Evaluation
}

\author{
Qiufang Wang and Yumei Shen \\ The Fourth Hospital of Baotou, Baotou 014030, China \\ Correspondence should be addressed to Yumei Shen; 102009178@btmc.edu.cn
}

Received 2 November 2021; Revised 24 November 2021; Accepted 25 November 2021; Published 22 December 2021

Academic Editor: Gengxin Sun

Copyright () 2021 Qiufang Wang and Yumei Shen. This is an open access article distributed under the Creative Commons Attribution License, which permits unrestricted use, distribution, and reproduction in any medium, provided the original work is properly cited.

\begin{abstract}
This paper combines flexible pressure sensing technology, wireless sensor network, and cloud platform technology to design and manufacture a medical miniature pressure sensor and its supporting system. The problem of noninvasive monitoring of the syndrome encountered in the clinic is used for real-time monitoring and auxiliary diagnosis of the disease. Different from the current clinical use of "puncture" to measure intrafascial pressure, this system focuses on the noninvasive monitoring of compartment syndrome, using medical tape to paste a flexible microsensing unit on the injured area. The flexible sensor unit can measure the pressure here in real time and then can know the pressure in the fascia chamber. The flexible pressure sensor unit combines with the subsequent flexible circuit to send the measured data to the data in real time through wireless communication. The data aggregation node transmits the collected data to the upper computer through serial communication, and the upper computer software processes and stores the data and uploads it to the cloud server. In this experiment, it was observed that the concentrations of $\mathrm{Ca}$ and $\mathrm{P}$ showed the same fluctuating trend. With the gradual progress of the stretch, the concentrations of $\mathrm{Ca}$ and $\mathrm{P}$ increased with the increase in time, reaching approximately at the end of the extension. The peak value indicates that the osteoclast activity is enhanced at this time, the bone matrix is largely destroyed, and the $\mathrm{Ca}$ and $\mathrm{P}$ in the matrix are released into the serum in a large amount, thereby increasing the serum concentration. After the distraction ceases, it enters the healing period of the callus. At this time, the concentrations of Ca and P decrease with the increase in time and gradually reach a stable level, indicating that the osteoblast activity is enhanced at this time, the bone matrix begins to rebuild, and the $\mathrm{Ca}$ and $\mathrm{P}$ gradually increase. The deposited bone matrix gradually forms new bone and finally reaches a balance. Since the speed of extension in each experimental group is inconsistent, the time required to reach the same extension length is also inconsistent, so that the peak time is also inconsistent. After plotting the stress difference $(\triangle F)$ before and after stretching against time and speed, it is found that the relationship is linear. However, these two variables affect $\triangle F$ at the same time, so they cannot be isolated. Based on this, this subject uses multiple regression equations to fit the three relationships of stress difference $(\triangle F)$, time, and speed. In the process of distraction osteogenesis, with each distraction, the bone stress presents a trend from high to low. And as the stretch progresses, the measured stress value increases linearly at the same time point every day.
\end{abstract}

\section{Introduction}

In orthopedics trauma, trauma caused by various sudden accidents, such as car accidents, slips, falls, and industrial accidents, may cause tissue damage or loss [1]. These injuries or defects often result in the loss of body functions and ultimately lead to disability or even death. Therefore, the care of wounds has attracted much attention from medical staff. Common traumas in orthopedics include open fractures, avulsions, extensive skin abrasions, and crush injuries. Currently, researchers and medical staff have developed many methods for treating wounds and repairing skin [2]. Among them, wound dressings are widely used for wound healing because they can provide suitable healing conditions and at the same time coordinate and balance body activities such as blood vessels, connective tissues, and epithelial cells. In addition, with the rapid development of China, the contradiction between the increasing aging population and the increasingly serious 
chronic diseases and China's relatively scarce medical resources has gradually become prominent [3]. At present, new concepts such as "smart medical" and "smart medical" are proposed to change the existing medical service model and improve the utilization of medical resources. Smart medical devices and wearable devices are an important part of these concepts. The research on sensors suitable for this type of equipment is of great significance to the promotion of "smart medical" and "smart medical." At the same time, this type of sensor device with the ability to perceive different external environmental parameters is also expected to be used in existing medical equipment such as artificial limbs to effectively improve the quality of life of patients [4].

When the external force is within the range of normal physiological load, in order to maintain its normal structure and function, the articular cartilage will obtain nutrients through the exchange of joint lubricating fluid and the fluid in the matrix. Therefore, normal joints usually do not cause cartilage damage when carrying loads within the bearing range, but any load that exceeds the joint bearing capacity has the risk of cartilage degeneration and osteoarthritis. For some athletes, soldiers or dancers, they often do some rapid acceleration, instant deceleration, continuous training and jumping and landing one-leg support. Therefore, due to their special activities, they may be greatly exacerbated by regular exercises in this way. The damage to the cartilage of the knee joint or the risk of worsening of the knee joint cartilage in people in China will have a serious impact on their careers. At present, for some different exercise methods, the human knee joint cartilage is subjected to different forms of loading conditions, and the mechanism of its mechanical properties has not been clearly expressed [5]. This requires us to strengthen the study of the changes in the mechanical behavior of the knee joint in different ways of movement and explore the inducing mechanism of the occurrence and exacerbation of knee cartilage damage under different loading conditions, as well as the changes in contact stress and mechanical behavior [6].

This paper designs the hardware of the flexible pressure sensor, which mainly includes the selection of the main components according to the system requirements, the design of the pressure signal conversion and reading circuit, the selection of the antenna, and the corresponding optimization processing. In the actual design, this article has been tightly focused on the requirements of flexibility, portability, and wearable. While satisfying the most basic functions of the system such as data reading and wireless communication, it can be achieved to the maximum extent. This subject observes that under the stretch strategy of extending twice a day, the stress value increases significantly after each stretch. As time goes by, this stress value gradually decreases until the next stretch (after 12 hours), approximately reaching the level before the last stretch. At this point, the second stretch is performed, and the stress value immediately increases and then gradually decreases with the passage of time. Until the first stretch on the next day, the stress value dropped to a slightly higher level than before the previous stretch. In this way, there are two stress peaks every day, and each peak appears at the moment after stretching and then gradually decreases with an exponential decay function. For each phase of distraction osteogenesis (the same number of days of extension), the experimental groups with different extension speeds showed different stress changes. The stress decreased by $8.83 \mathrm{~N}$ before and after the first stretch on the first day, and the stress increased by $12.52 \mathrm{~N}$ before and after the second stretch and then dropped by $11.21 \mathrm{~N}$.

\section{Related Work}

Combining the patient's clinical manifestations, imaging examinations, serological indicators, infrared spectroscopy, and direct manometry are the main methods for diagnosing acute compartment syndrome. Pain, paleness, paresthesia, pulselessness, and paralysis are the five main clinical manifestations. Serological indicators are mainly accelerated erythrocyte sedimentation rate, increased white blood cell count, and increased creatine kinase. The first two are mainly caused by the body under stress conditions, and the increase in the two can appear under many stress conditions, lacking specificity. Creatine kinase is of relatively great value in the diagnosis of compartment syndrome. Normally, there is a small amount of this enzyme in serum, 25-170 U/L for women and 25$200 \mathrm{U} / \mathrm{L}$ for men. It is an important enzyme that regulates cell energy metabolism. When the body is severely traumatized or compartment syndrome occurs, resulting in muscle ischemia and necrosis, the enzyme in the cell will be released into the blood, which will significantly increase the content of serum creatine kinase. It began to rise sharply 2 hours after injury and reached a peak at 24 hours after injury, which was 42 times the normal value.

Studies by related scholars have reported that when muscle tissue develops edema and degeneration, the average creatine kinase value reaches $2400 \mathrm{U} / \mathrm{L}$ or more; the osteofascial compartment should be cut and decompressed at this time [7]. At this time, the nerve, blood vessel, and muscle ischemia time is not yet very long, without avascular necrosis, causing irreversible damage. MRI examination can mainly see manifestations such as increased volume of the osteofascial compartment, muscle edema, and loss of muscle texture. Doppler ultrasound is more valuable in the diagnosis of compartment syndrome than other auxiliary examinations. It can not only observe tissue edema and vessel diameter, but also dynamically observe arterial pulsation and fascial tissue elasticity changes. Related scholars have studied the pulsed phase-locked loop ultrasound instrument to observe the fascia displacement waveform of the arterial blood pressure pulse to estimate the pressure of the osteofascial compartment [8]. The correlation between the degree of fascia displacement in the osteofascial compartment and the perfusion pressure was confirmed by the researchers through noninvasive ultrasound. Relevant scholars have established a model of the anterior tibial compartment of the calf to study the increased pressure of the fascial compartment (infusion of $0.9 \%$ sodium chloride solution increases the pressure of the compartment) [9]. The reduced feasibility of noninvasive evaluation has made Doppler ultrasound more and more widely used in the diagnosis of compartment syndrome. 
Related scholars have studied the creep behavior of articular cartilage and the mechanical behavior of depth and velocity correlation [10]. It is found that under different creep times of articular cartilage, the creep strain and creep compliance decrease along the depth of the cartilage from the surface to the depth. The depth-dependent creep compliance increases with the creep time, and the increase in creep compliance decreases along the cartilage depth. As the creep progresses, the creep compliance first increases rapidly, then increases and slows down, and decreases as the compressive stress increases. In addition, Young's modulus will gradually increase from the surface layer to the deep layer, and Young's modulus of different layers will increase with the increase in the stress rate [11]. Under the set compressive strain, the Poisson's ratio will increase with the increase in depth and the Poisson's ratio of different layers will increase with the increase in compressive strain.

Related scholars have studied the dynamic contact mechanics of the human knee tibial platform during gait and stair climbing, analyzed the magnitude and position of the maximum contact stress at different relative positions during the movement [12]. The maximum contact stress occurs in the cartilage-cartilage contact area. During climbing stairs, the maximum stress occurs on the back of the platform under the meniscus; in the early stages of gait and ladder climbing, the maximum stress on the lateral platform appears under the meniscus. At the later stage of the ladder climbing process, the maximum contact stress appears in the cartilage-cartilage contact area. Related scholars have studied the stress contact mode on the tibial plateau during the simulated gait movement [13]. The first mode occurs on the posterior side of the tibial plateau and a single peak stress occurs in the early stage; the second mode occurs in the middle and posterior part of the lateral plateau, with two peaks in the early and late stages; the third mode occurs on the medial plateau cartilage.

Relevant scholars have studied the changes of some knee joint motion parameters during walking before and after a period of kneeling position and found that the continuous static load on the knee joint will significantly affect the subsequent knee joint motion load pattern [14-16]. The motion and work style of static load applied to the joint will obviously affect the force of the knee joint movement, which will cause or aggravate the risk of arthritis [17, 18]. The researchers found through finite element simulation that even when the damage occurs only through strain-related damage mechanisms, the location and size of cartilage damage are obviously dependent on the strain rate [19-21]. In addition, experimental studies have found that for a given compression amplitude of up to $1.2 \mathrm{~mm}$, the reaction force changes 6 times in the compression rate [22-24]. Although the static response is basically linear, the nonlinear behavior increases with the increase in the compression rate $[25,26]$.

\section{Method}

3.1. Flexible Pressure Sensor Terminal Architecture. The hardware part of the flexible pressure sensor mainly includes a flexible pressure sensing unit module, a pressure data reading and conversion module, a wireless communication module, and a power supply module. The overall hardware architecture is shown in Figure 1. The flexible pressure sensing unit converts the pressure signal into the resistance (conductance) signal, pressure data reading and conversion module reads and converts the resistance signal into a digital signal and sends it to the wireless communication module. The wireless communication module sends the data to the data aggregation node through the ZigBee wireless communication protocol, the data is transmitted to the upper computer by the transmission method, the upper computer software further processes the data and stores and uploads the data, and finally the power module is responsible for supplying power to the entire flexible pressure sensor.

3.2. Flexible Pressure Sensing Unit. The flexible pressure sensing unit is a flexible pressure sensing device, which has many advantages such as good flexibility, free bending and even folding, and being light and thin, portable, and wearable. It is very suitable for pressure data measurement on wearable devices. This article analyzes the advantages and disadvantages of the three mainstream flexible pressure sensing units: piezoelectric flexible pressure sensing unit, capacitive flexible pressure sensing unit, and piezoresistive flexible pressure sensing unit. Aimed at the scenario of comprehensive monitoring of the osteofascial compartment, this paper intends to use a piezoresistive flexible pressure sensing unit as the pressure acquisition device of the system.

The flexible pressure sensing unit used in this article is Flexiforce from Tekscan, USA. The thickness of the flexible pressure sensing unit is only $0.15 \mathrm{~mm}$, and the diameter of the sensitive area is $0.95 \mathrm{~cm}$. The range of different types of flexible pressure sensing units can range from a few Newtons to hundreds of Newtons, which can be used by different users $[27,28]$. Flexiforce is very flexible, can be bent at will like paper, and is very thin, which fits the application scenarios of compartment syndrome pressure measurement in this article.

The Flexiforce flexible pressure sensing unit adopts a two-layer structure, each layer of film is made of polyester fiber material, and then silver wires are printed on this layer of film, and finally a layer of pressure sensitive material is added, and the two films are glued together. The mixture is pasted together to form the Flexiforce flexible pressure sensing unit. When no pressure is applied to the sensitive area, the Flexiforce flexible pressure sensing unit presents a high resistance state; that is, the resistance of the Flexiforce flexible pressure sensing unit is infinite, usually on the order of tens of megaohms. When a force is applied to the sensitive area, the resistance of the Flexiforce flexible pressure sensor unit will drop to the order of tens of kiloohms; that is, the resistance of the Flexiforce flexible pressure sensor will decrease as the pressure increases, and the conductance increases with the pressure.

The conductance of the Flexiforce flexible pressure sensing unit is related to the pressure; that is, there is a one-toone correspondence between the pressure and the conductance. However, since each sample of the Flexiforce flexible pressure sensing unit is not exactly the same $[29,30]$. Therefore, it is necessary to calibrate the pressure conductance 


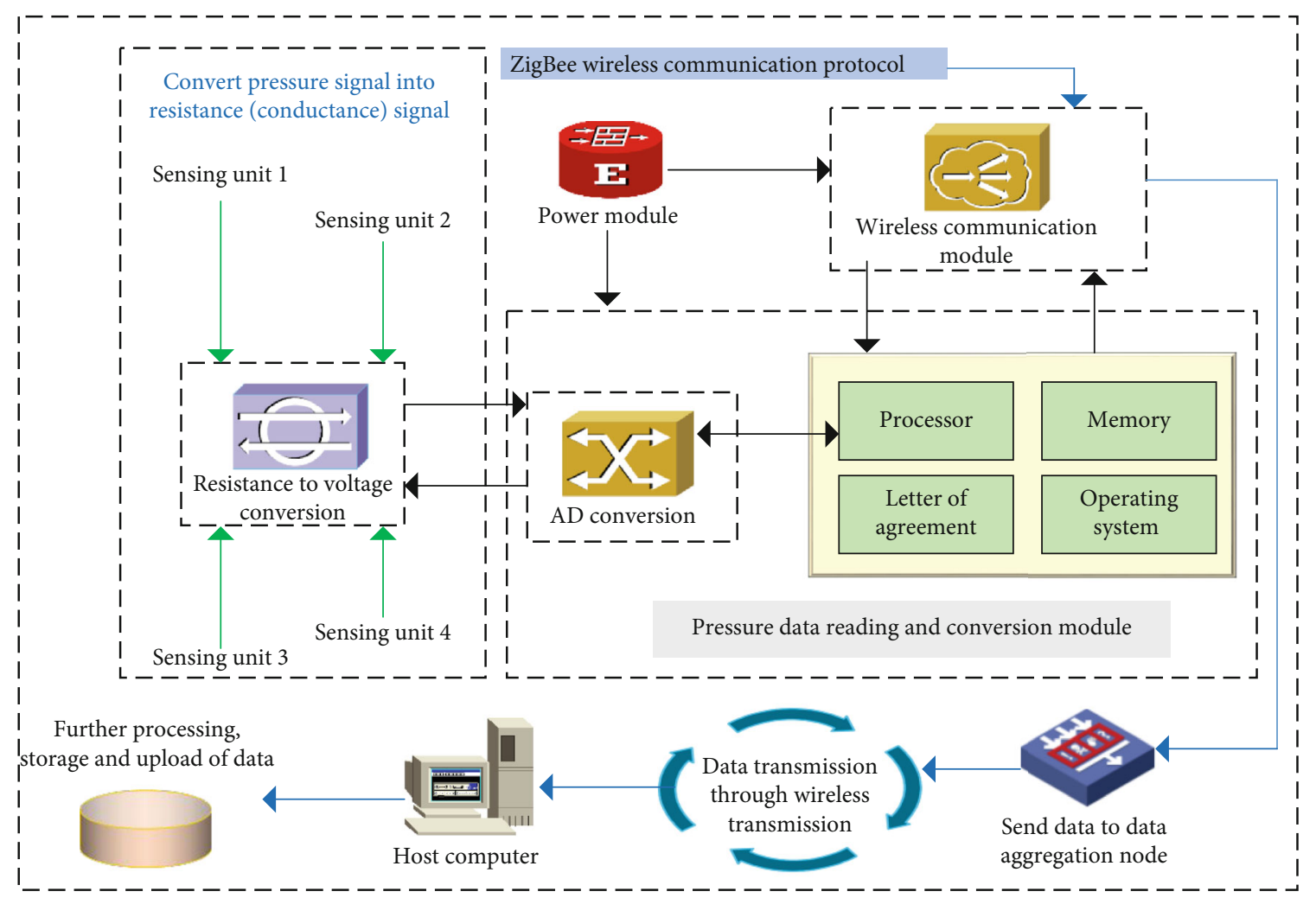

Figure 1: The overall architecture of flexible pressure sensor terminal hardware.

curve of each Flexiforce flexible pressure sensing unit sample and do the corresponding recording and storage work to process the data later.

Place the Flexiforce flexible pressure sensing unit on the rotating screw lifting platform to ensure that the sensitive unit is located directly under the push-pull force gauge. The two electrodes of the pressure sensing unit are, respectively, connected to the red and black test leads of the multimeter to measure the resistance value, and the pressure conductivity data is recorded within the pressure range of the Flexiforce flexible pressure sensing unit. Figure 2 is the pressure conductance curve of the Flexiforce flexible pressure sensing unit with a range of $5 \mathrm{~N}$. It can be seen that the flexible pressure sensing unit has good repeatability within its range.

3.3. ZigBee Wireless Communication. This article intends to use ZigBee as the wireless communication protocol of the auxiliary monitoring system for compartment syndrome. There are currently four mainstream ZigBee wireless communication chips on the market: Texas Instrument (TI) CC2530, SILABS EM35x, FREESCALE MC13224, and JENNIC's JN516x. The parameters of the four chips are shown in Table 1.

According to the parameters in Table 1, combined with our application scenario of compartment syndrome, this paper intends to use the CC2530 with a smaller chip size and a lower price as the wireless communication chip of this system. CC2530 is the second-generation ZigBee wireless communication chip launched by TI. The wireless communication chip works in the $2.4 \mathrm{GHz}$ frequency band and inte- grates modules such as a microcontroller, wireless communication, and analog-to-digital converters. CC2530 adopts the QFN40 package, the physical size is only $6 \mathrm{~mm}$ $* 6 \mathrm{~mm}$, the input voltage is between $2 \mathrm{~V}$ and $3.6 \mathrm{~V}$, and the sending and receiving data currents are relatively small, $24 \mathrm{~mA}$ and $29 \mathrm{~mA}$, respectively.

The following mainly introduces the modules contained in the chip:

(1) Microcontrol unit: the wireless communication chip contains an enhanced 8051 microcontrol unit with three different memory access bus modes: special function registers, DATA, and CODE/XDATA. In addition, it also includes a debug interface and an input expansion interrupt unit

(2) Flash memory: the flash memory of the CC2530 is mainly used to retain program codes and constants. There are 4 different versions of the chip, which are mainly distinguished according to the memory size. The memory size is divided into $32 \mathrm{~KB}, 64 \mathrm{~KB}$, $128 \mathrm{~KB}$, and $256 \mathrm{~KB}$

(3) Random access memory: this module can retain data when the digital part is powered down, reducing the power consumption of the entire system

(4) Analog-to-digital converter: ADC is 12 bits, supports 8 channels of analog signal input, and can choose single-ended or differential input 


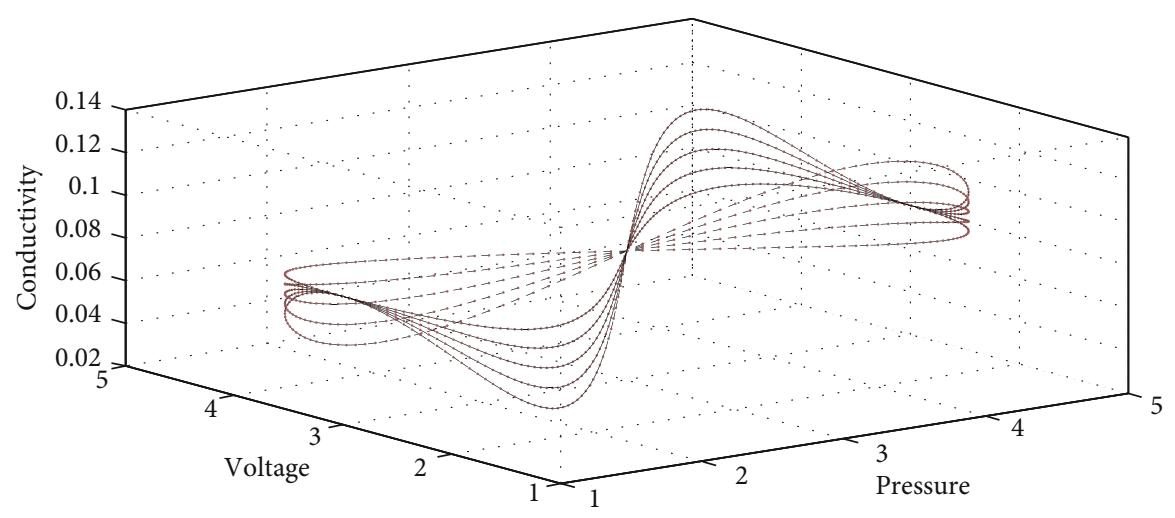

Figure 2: Flexiforce pressure conductance.

TABLE 1: ZigBee chip parameter table.

\begin{tabular}{lcccc}
\hline Chip model & MC13224 & JN516x & CC2530 & EM35x \\
\hline Size & $9.5 \mathrm{~cm} * 9.5 \mathrm{~cm}$ & $5 \mathrm{~cm} * 5 \mathrm{~cm}$ & $7 \mathrm{~cm} * 7 \mathrm{~cm}$ & $8.2 \mathrm{~cm} * 8.2 \mathrm{~cm}$ \\
Maximum transmit power & $4.2 \mathrm{dBm}$ & $2.4 \mathrm{dBm}$ & $5.4 \mathrm{dBm}$ & $9.4 \mathrm{dBm}$ \\
Received power & $-91 \mathrm{dBm}$ & $-95 \mathrm{dBm}$ & $-98 \mathrm{dBm}$ & $-95 \mathrm{dBm}$ \\
Tx current & $16 \mathrm{~mA}$ & $14 \mathrm{~mA}$ & $27 \mathrm{~mA}$ & $19 \mathrm{~mA}$ \\
Rx current & $18 \mathrm{~mA}$ & $16 \mathrm{~mA}$ & $22 \mathrm{~mA}$ & $29 \mathrm{~mA}$ \\
Lowest power consumption current & $0.33 \mu \mathrm{A}$ & $0.11 \mu \mathrm{A}$ & $0.51 \mu \mathrm{A}$ & $0.43 \mu \mathrm{A}$ \\
\hline
\end{tabular}

(5) Watchdog: the watchdog plays the role of protecting the microcontroller, resetting the device, and waking up the device from sleep state when the software fails

(6) Sleep mode timer: this timer is an ultralow power timer with a $32 \mathrm{kHz}$ frequency crystal oscillator, which can be used as a sleep wake-up timer

(7) Wireless transceiver module: this module includes an IEEE 802.15.4 radio frequency transceiver, data packet filtering, and address recognition module

3.4. Wireless Pressure Sensor Assistance. The main function of the flexible pressure sensor is to collect pressure information at the wound of compartment syndrome. The pressure is not an electrical quantity. We are not good at measuring and processing it directly. Therefore, the pressure change needs to be converted through the flexible pressure sensing unit. It is the resistance change, and then the resistance change is converted into a voltage change. In this way, we can measure and process it and finally send the data to the data aggregation node in the data center through wireless communication. The data aggregation node then transmits the pressure data to the upper computer through serial communication, and the software of the upper computer displays, graphs, stores, and uploads the data to the cloud, so as to achieve the purpose of real-time monitoring of pressure information at the wound of compartment syndrome.

The resistance of the sensitive area of the Flexiforce flexible pressure sensing unit is inversely proportional to the pressure. The greater the pressure, the lower the resistance of the Flexiforce flexible pressure sensing unit. For the mea- surement of resistance change, this article uses the same phase amplifier circuit to achieve. According to the knowledge in analog electronic circuits, it can be known from the "virtual short" and "virtual disconnection" that the input voltage and output voltage of the same phase amplifier circuit have the following relationship:

$$
V_{\text {out }}=\left(\frac{1-R_{x}}{R_{\text {ref }}}\right) \bullet\left(R_{\text {ref }} \bullet V_{\text {in }}\right),
$$

where $V_{\text {in }}$ is the input voltage, $V_{\text {out }}$ is the output voltage, $R_{\text {ref }}$ is the reference resistance, and $R_{x}$ is the resistance of the piezoresistive flexible pressure sensing unit. When $V_{\text {in }}$ and $R_{\text {ref }}$ are constant, the output voltage $V_{\text {out }}$ of the circuit is inversely proportional to the resistance $R_{x}$ of the piezoresistive flexible pressure sensor, that is, proportional to its conductance. According to the previous content, the conductance of the piezoresistive flexible pressure sensor is proportional. Pressure is positively correlated and can be fitted with a linear function, so the output voltage of this circuit has a linear function relationship with pressure.

$$
V_{\text {out }}=\frac{C_{1} S_{x}}{R_{x}}+C_{2} R_{x}-C_{3}(1-F)+C_{4} .
$$

In the formula, $S_{x}$ is the conductance of the Flexiforce flexible pressure sensing unit, $F$ is the pressure on the sensitive area of the Flexiforce flexible pressure sensing unit, and $C_{1}$, $C_{2}, C_{3}$, and $C_{4}$ are constants. 


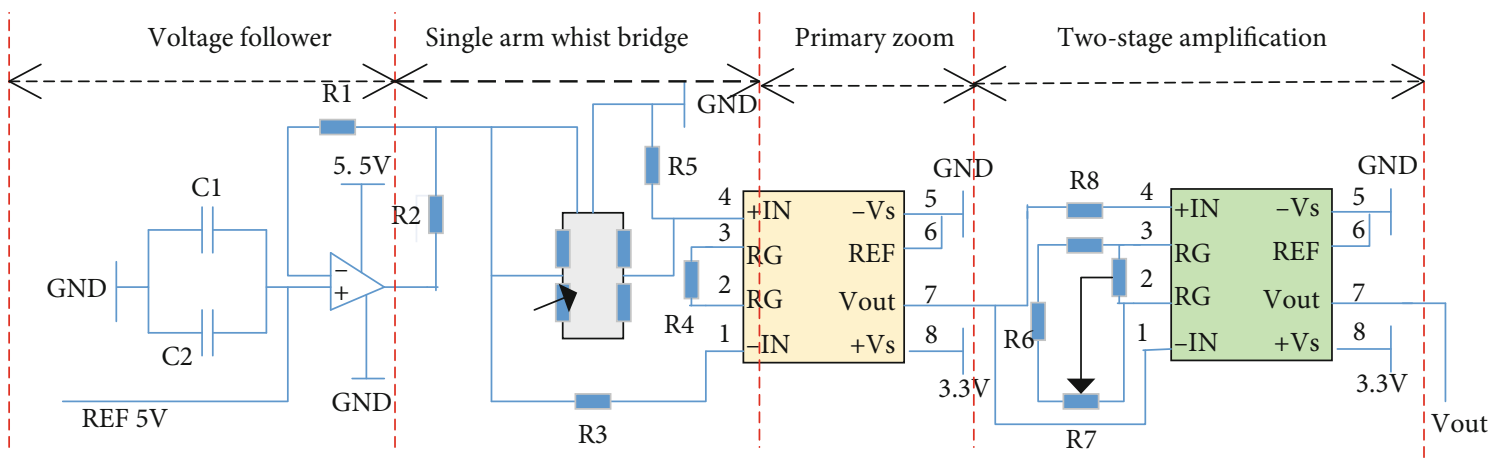

FIgURE 3: Two-stage amplifying circuit composed of AD8236.

The power module is one of the key modules of the flexible pressure sensor. The CC2530 chip, the operational amplifier chip (MCP6004), and the JTAG (Joint Test Action Group) interface in the flexible pressure sensor all require a stable $3.3 \mathrm{~V}$ power supply. Although there are many $3.3 \mathrm{~V}$ batteries to choose from, most of these batteries on the market have a wide range of variations and are not very stable $3.3 \mathrm{~V}$ voltages. For example, some $3.3 \mathrm{~V}$ lithium batteries have a voltage of $4.2 \mathrm{~V}$ when fully charged. The latter voltage becomes about $2 \mathrm{~V}$, so the power supply voltage by a battery is usually unstable. And we need a stable $3.3 \mathrm{~V}$ power supply. If the power supply voltage is unstable, it is likely to affect the conversion accuracy of the internal analog-to-digital conversion module of the CC2530 chip and even affect the normal operation of the device, so the basic design idea of the module is $3.7 \mathrm{~V}$. The output is followed by a low dropout voltage regulator chip (low dropout voltage (LDO)). Through such processing, a stable $3.3 \mathrm{~V}$ voltage is provided to the CC2530 chip and the operational amplifier chip. In order to reduce the size of the flexible pressure sensor power supply module as much as possible, this design uses a $3.7 \mathrm{~V}$ rechargeable battery with a capacity of $60 \mathrm{mAh}$. The size of the battery is $15 \mathrm{~mm} * 12 \mathrm{~mm} * 4 \mathrm{~mm}$ in length.

The signal amplification link uses the instrumentation amplifier AD8236 as the core chip. This amplifier is an instrumentation amplifier with low price, low power consumption, and output swing that can reach the power supply voltage (usually called power limit output). It has been widely used in microcurrent detection, portable equipment, and medical equipment. The input range of the sensing signal is $0 \sim 3.3 \mathrm{mV}$, and the voltage input range of the analog-digital converter $(\mathrm{ADC})$ that needs to be connected to the microcontroller is $0 \sim 3.3 \mathrm{~V}$; then, the required gain is $\beta=1000$. This article uses two-stage amplification to meet the amplification requirements, and the corresponding circuit is shown in Figure 3. The power supply voltage of the system is provided by a $5 \mathrm{~V}$ DC-DC DC power supply circuit. A voltage follower is added to the input of the amplifier to improve the ability of the reference voltage to carry a load and to isolate interference from other modules. In order to calibrate that the input of the amplifying circuit is zero when the sensor is not working, an adjustable resistor and a zero-adjusting circuit are added before the two-stage input of the amplifying circuit.
3.5. Analysis of Error Sources. The contact pressure signal will be affected by environmental factors (such as temperature) during the process from acquisition to amplification, causing measurement errors. It is necessary to analyze the sources of system errors. In the Wheatstone bridge link, the main factor that affects the change in the output voltage of the bridge is temperature. The change of temperature will cause the sensitivity coefficient $K$ of the strain gauge to change. According to the calculation formula of the bridge output voltage, when the temperature changes by $\Delta T$, the output voltage of the bridge is

$$
U_{\mathrm{To}}=K \frac{\left(1-\varepsilon_{L}\right)\left(1-\alpha_{k} \Delta T\right)}{(K+1) \varepsilon_{L} U_{i}\left(1+2 \alpha_{k} \Delta T\right)} .
$$

In the formula, $\alpha_{k}$ is the sensitivity temperature coefficient of the strain gauge.

In the signal amplification link, the amplifier is a lowspeed and high-precision application in the environment described in this article. It is necessary to focus on lowfrequency DC errors, such as low-frequency noise, bias current, and offset voltage. The error sources of the gain $\beta$ of the amplifier used in the amplifying link include the temperature of the environment (or the measured object), the environmental noise, the voltage and current noise of the amplifying chip, and the offset voltage. Among them, the external environmental noise is extremely small in ordinary environments, usually below $0.1 \%$, and the internal noise of the amplifier itself is in the $\mu \mathrm{Vp}$-p level, so the influence of these two items on the amplified output voltage is negligible.

The absolute error part of the amplifier mainly includes gain error $S_{\beta}$, common mode error $S_{\mathrm{CMR}}$, and output offset voltage error $S_{a o}$. The temperature drift error is divided into gain drift and input offset voltage drift (ignoring input offset current drift). Combining the errors of these two parts, the expression of the sum of errors $S_{a}$ of the amplification link can be obtained as follows:

$$
S_{a}=\left(1-\alpha_{a t}\right) \Delta T-\alpha_{a o} S_{\beta}+S_{a o} S_{\mathrm{CMR}}
$$

In the formula, the units of $S_{\beta}, S_{\mathrm{CMR}}$, and $S_{a o}$ are ppm, and $\alpha_{a t}$ and $\alpha_{a o}$ are the temperature coefficients of gain and offset 


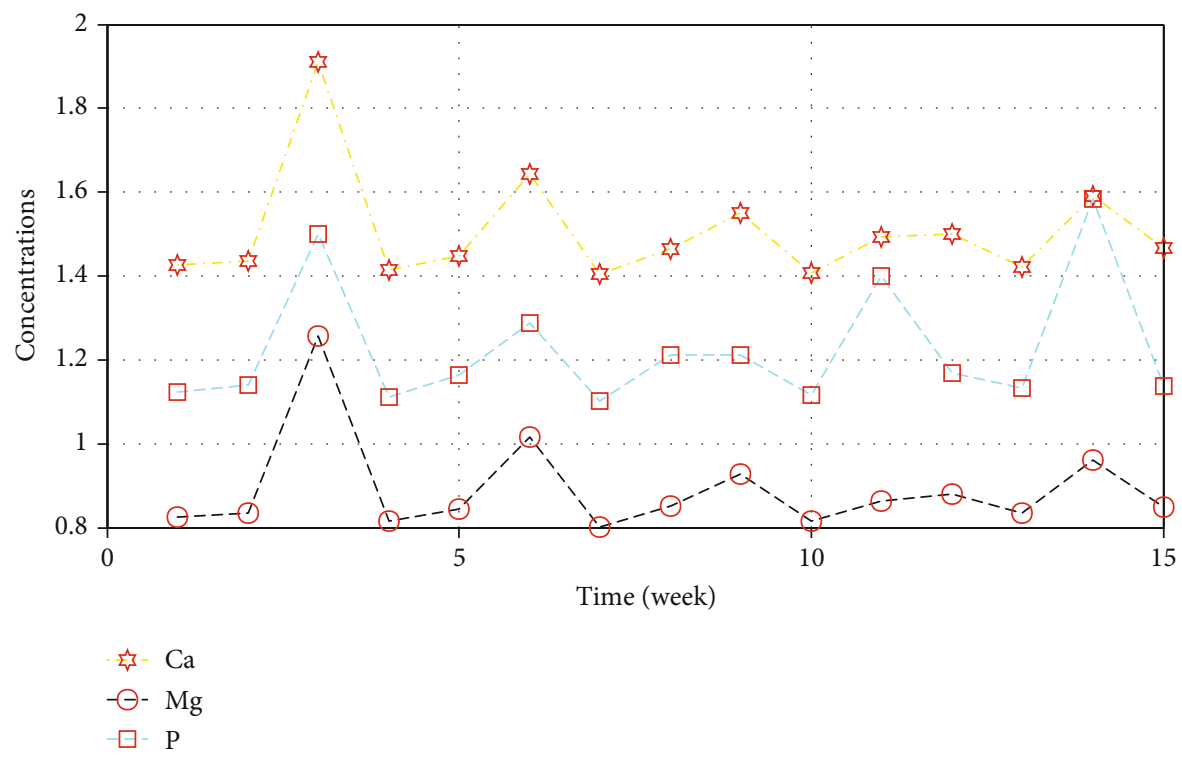

Figure 4: The change trend of serum $\mathrm{Ca}, \mathrm{Mg}$, and $\mathrm{P}$ concentrations with the progress of bone elongation.

voltage (in $\mathrm{ppm} /{ }^{\circ} \mathrm{C}$ ), respectively. For the $\mathrm{DC}$ amplifier circuit, the main source of error is the static error part (such as offset error), which can be eliminated by calibration.

When considering the temperature change, it is necessary to compensate for the error caused by the temperature drift. There are many methods of temperature compensation, which can be divided into two types: self-compensation and bridge compensation. Among them, the self-compensation method is to make the additional strain of the strain gauge zero by selecting the appropriate sensitive grid material and structural parameters when the linear expansion coefficient of the material to be tested is known, so that the resistance change caused by the temperature change is also zero, in order to achieve the purpose of temperature compensation. Although this compensation method can realize zero resistance change caused by temperature from the perspective of resistance deformation, it needs to know the temperature coefficient of the tested piece and other parameters, so there will be certain errors in actual use. The compensation effect of the occasion is not ideal.

The bridge compensation method uses other arms in the full bridge as reference strain gauges, which are exactly the same as the working strain gauge model parameters and are all attached to the test piece in the same temperature environment, but do not bear the effect of strain. Then, the bridge is still in equilibrium when the temperature changes, and the change in output voltage is only related to the pressure on the working strain gauge. The effect of bridge compensation is better than self-compensation, so it is also a more commonly used compensation method in engineering applications.

In a single-arm bridge, the bridge compensation method can be used for temperature compensation. $R_{1}$ is the working strain gauge, then $R_{2}$ should be selected as the reference strain gauge, and the other arms are matched resistances. The resistance change of $R_{1}$ under the action of temperature and pressure is $d R_{P}$ and $d R_{T}$, respectively. $R_{2}$ is only affected by temperature, and its resistance change is $d R_{T}$. Then, the output voltage of the bridge is

$$
U_{o}=\left[\frac{R_{1}+d\left(R_{p}-R_{T}\right)}{R_{1}-R_{2}+(1-d)\left(R_{p}-R_{T}\right)}-\frac{R_{2}}{R_{p}-R_{3}}\right] U_{i} .
$$

Choosing the initial resistance $R_{1}=R_{2}=R_{3}=R_{4}=R$, we can get

$$
\frac{U_{o}}{U_{i}}=\frac{d R_{T}\left(R_{p}+d R\right)}{3\left(R_{p}-d R\right)-d R_{T}} .
$$

For the resistance change caused by pressure in the same temperature environment, we can get

$$
\frac{U_{o}}{U_{i}}=\frac{(1-K) \varepsilon_{L}}{3 \varepsilon_{L}-K R_{T}} .
$$

It can be seen from the above formula that the change in the resistance of the strain gauge caused by the temperature is offset in the bridge, and the output of the bridge is only related to the input voltage and strain. It should be noted that the premise of using this temperature compensation method is that the parameters of the working strain gauge and the reference strain gauge are exactly the same, the installation conditions are the same, and the resistance values of the other two arms always remain unchanged during temperature changes.

\section{Result Analysis}

4.1. Determination of Biochemical Indicators of Bone Metabolism. In this subject, various bone metabolism-related indicators in the serum of experimental samples in different experimental processes were determined. The main biochemical indicators measured include $\mathrm{Ca}, \mathrm{Mg}$, and $\mathrm{P}$. In the 




Figure 5: The edge extraction of the anterior and lateral positions of the film after the operation.



FIGURE 6: Mechanical properties of bone bending after elongation stops.

abscissa, "1-15" are the samples collected on the first day of the 1-15 weeks after surgery. According to the stretch strategy, extension is only performed one week after surgery.

It can be seen from Figure 4 that the concentrations of $\mathrm{Ca}, \mathrm{Mg}$, and $\mathrm{P}$ show similar fluctuation trends. Stretching was performed one week after the operation. With the gradual progress of the stretch, the concentrations of $\mathrm{Ca}, \mathrm{Mg}$, and $\mathrm{P}$ increased with time, reaching a peak at about the third week, after which the stretch stopped and the callus healing period entered. At this time, the concentration of $\mathrm{Ca}, \mathrm{Mg}$, and $\mathrm{P}$ fluctuates with the increase in time and gradually stabilizes. Since the speed of extension in each experimental group is inconsistent, the time required to reach the same extension length is also inconsistent, making the time of peak appearing in the graph also inconsistent.

4.2. X-Ray Judgment of Bone Healing. After the osteotomy was completed, X-ray monitoring of the surgical site was performed. The result is shown in Figure 5. It can be seen from Figure 5 that the fractured ends of the bone can be clearly seen on the X-ray film and the fracture line is clear; there is a zona pellucida of about $0.5 \mathrm{~mm}$ between the fractured ends (pointed by the red arrow), which is the stable fixation of the external fixation device. Regardless of whether it is in the frontal or lateral position, the alignment and alignment of the bone ends are good.

4.3. Mechanical Property Test at the Later Stage of Bone Healing. When analyzing the mechanical properties of the bone after the extension is stopped, if the material is defined as a pipe, the bending strength can be increased to $400 \mathrm{MPa}$. At the same time, the elastic modulus has also increased from $20 \mathrm{MPa}$ to $140 \mathrm{MPa}$. Such a large difference in elastic modulus shows that in the same sample test, the setting of material properties in the experiment has a great influence on the results. Therefore, in this experiment, the material is set as the pipe material, and the experimental group and the control group use the same pipe diameter setting. Healed bone has strong plasticity and weaker brittleness, so it can be inferred that the bone tissue is still in the mid-healing stage at this time and cannot bear a large load. The mechanical 
TABLE 2: Test results of bone mechanical properties 2 weeks after stopping lengthening.

\begin{tabular}{lcccccc}
\hline Sample & Material & Elastic modulus & Bending strength & Breaking strength & Sample area & Maximum load \\
\hline \multirow{2}{*}{ Test } & Bar & 13 & 23 & 21 & 165 & 145 \\
& Pipe & 176 & 433 & 335 & 95 & 112 \\
\multirow{4}{*}{ Control } & Bar & 15 & 178 & 147 & 161 & 1178 \\
& Pipe & 1165 & 4556 & 3789 & 98 & 1177 \\
\multirow{2}{*}{ Ratio } & Bar & 82 & 14 & 13 & 0.98 & 12 \\
& Pipe & 15 & 8 & 8.7 & 0.99 & 9 \\
\hline
\end{tabular}

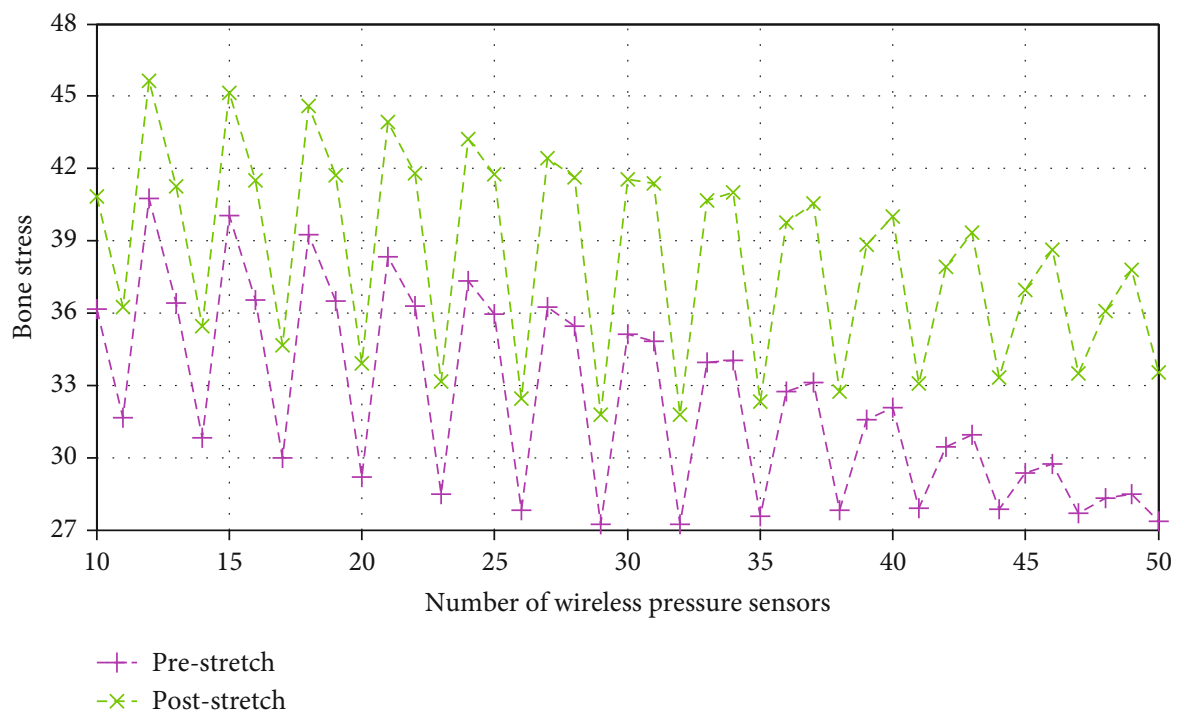

FIGURE 7: The change trend of bone stress value with the number of wireless pressure sensors.



Figure 8: The 3D trend graph of $\triangle F$ changes with time and stretch speed.

properties of bone bending after the extension stops are shown in Figure 6.

It can be seen from Table 2 that in the analysis of the comparative bone mechanical properties, if the material is defined as a tube, the bending strength of the healing bone is only $184.85 \mathrm{MPa}$, and if the material is defined as a tube, the bending strength can be increased to $4,594.56 \mathrm{MPa}$. The modulus of elasticity is $1170.84 \mathrm{MPa}$. The healed bone is more rigid and less brittle. Compared with the healing bone in the experimental group, the elastic modulus of the control group is significantly higher than that of the experimental group, so the rigidity of the former is much greater than that of the latter.
4.4. Determination of Bone Stress Level. With the passage of time, this stress value gradually decreases and reaches the level before the last stretch before the next stretch. At this point, the second stretch is performed, and the stress value immediately increases and then gradually decreases with the passage of time. Until the first stretch on the next day, the stress value dropped to slightly higher than the level before the previous stretch. In this way, there are two stress peaks every day, and each peak appears at the moment after stretching and then gradually decreases with an exponential decay function. The stress value at the initial stage of decline decreases rapidly, and this speed gradually slows down. This 
process just coincides with the exponential decay function model. The change trend of bone stress value with the number of wireless pressure sensors is shown in Figure 7.

The 3D trend graph of $\triangle F$ changing with time and stretching speed is shown in Figure 8 . Since $\triangle F$ also changes with the change of the stretching speed, the stress-time curve alone is not enough to explain the change trend of the stress difference $(\triangle F)$. The previous experiment proved that at the same time point, the stress difference $(\triangle F)$ is also linear with different stretching speeds, so we used multiple linear regression fitting to simultaneously investigate the influence of two variables on $\triangle F$.

\section{Conclusion}

The results of this experiment show that after stopping distraction for 2 weeks, the torsion resistance and bending resistance of the healed bone are lower than those of the healthy side control group. In particular, the bending strength and breaking strength of the healed bone are only $10 \%$ of the control group. The elastic modulus is about $15 \%$ of the control group. The torsion strength, yield strength, yield shear stress, maximum shear stress and other indicators of the healing bone were only about $50 \%$ of the control group. This shows that the healing bone is in the early stage of secondary healing at this time, and the plasticity of the healing bone is strong but the rigidity is weak. If the external fixator is removed at this time, it is very prone to secondary fractures. After stopping the distraction for 10 weeks, the plasticity of the healing bone increased significantly compared with that at 2 weeks, especially the fracture strength was close to the normal bone tissue (95.4\%), and there was no significant difference between the healing bone and the healthy side bone $(p>0.01)$. However, the elastic modulus is still quite different from the normal control (27.2\%). Therefore, it can be inferred that the bone is already at the end of the healing period and can bear a larger load. The later recovery is mainly the recovery of the elastic modulus. As far as torsion strength is concerned, there is no significant difference between the experimental group and the control group $(p>0.01)$. The difference in shear modulus is $14 \%$. The maximum shear stress of the healing bone is slightly higher than that of the control, and the yield shear stress is slightly lower than that of the control, indicating that the healing degree of the healing bone can bear the corresponding shear stress, but it will yield early. This suggests that its plasticity is slightly higher than its own control. It will take some time for the bone tissue to heal completely. Comprehensive analysis shows that the healing bone is currently at the end of the second stage of healing, and its callus strength has been able to withstand its own gravity and has a certain degree of resistance to torsion and bending. At this time, it is completely feasible to remove the external fixator. This subject has greatly controlled the weight difference when grouping, but later found that there are differences in the thickness of bones of the same weight, and whether these differences will also have a greater impact on the change in stress value $(\triangle F)$. The next step in this topic will be to explore other factors that affect bone stress changes in order to establish an equation based on multiple parameters to prepare for preclinical experiments.
In the research at this stage, the system construction and performance testing and optimization of the bone stress monitoring device have been completed, and the bone stress change curve $(\triangle F)$ based on the binary regression equation has been initially established for the in-depth study of bone stress and bone healing. The relationship has laid a solid experimental foundation.

\section{Data Availability}

The data used to support the findings of this study are available from the corresponding author upon request.

\section{Conflicts of Interest}

The authors declare that they have no known competing financial interests or personal relationships that could have appeared to influence the work reported in this paper.

\section{References}

[1] P. Avaltroni, S. Nappi, and G. Marrocco, "Antennifying orthopedic bone-plate fixtures for the wireless monitoring of local deep infections," IEEE Sensors Journal, vol. 21, no. 18, pp. 21012-21021, 2021.

[2] P. M. S. Shimabukuro, M. L. Duarte, A. M. Imoto et al., "Environmental cleaning to prevent COVID-19 infection. A rapid systematic review," Sao Paulo Medical Journal, vol. 138, no. 6, pp. 505-514, 2020.

[3] S. I. Jeong, E. J. Lee, G. R. Hong et al., "Three-dimensional multistack-printed, self-powered flexible pressure sensor arrays: piezoelectric composites with chemically anchored heterogeneous interfaces," ACS Omega, vol. 5, no. 4, pp. 19561965, 2020.

[4] Y. Xiao, Y. Duan, N. Li et al., "Multilayer double-sided microstructured flexible iontronic pressure sensor with a recordwide linear working range," ACS Sensors, vol. 6, no. 5, pp. 1785-1795, 2021.

[5] A. Palmroth, T. Salpavaara, P. Vuoristo et al., "Materials and orthopedic applications for bioresorbable inductively coupled resonance sensors," ACS Applied Materials \& Interfaces, vol. 12, no. 28, pp. 31148-31161, 2020.

[6] S. Li, F. Hu, Z. Xu, Z. Mao, Z. Ling, and H. Liu, "Joint power allocation in classified WBANs with wireless information and power transfer," IEEE Internet of Things Journal, vol. 8, no. 2, pp. 989-1000, 2020.

[7] R. Saidi, H. Heidari, M. Sedehi, and B. Safdarian, "Evaluating the effect of Matricaria chamomilla and Melissa officinalis on pain intensity and satisfaction with pain management in patients after orthopedic surgery," Journal of Herbmed Pharmacology, vol. 9, no. 4, pp. 339-345, 2020.

[8] X. Liu, L. Li, L. Wang, K. Herr, and Q. Chen, "Implementation and evaluation of a pain management core competency education program for surgical nurses," International Journal of Nursing Sciences, vol. 8, no. 1, pp. 51-57, 2021.

[9] Y. Li, W. Chen, and L. Lu, "Wearable and biodegradable sensors for human health monitoring," ACS Applied Bio Materials, vol. 4, no. 1, pp. 122-139, 2020.

[10] M. Beccatelli, M. Villani, F. Gentile et al., "All-polymeric pressure sensors based on PEDOT: PSS-modified polyurethane 
foam," ACS Applied Polymer Materials, vol. 3, no. 3, pp. 15631572, 2021.

[11] P. W. K. Putra, I. K. A. Widiantara, and A. A. N. Kusuma, "Effectiveness of the use of acupressure wristband at Neiguan point (P 6) towards postoperative nausea vomiting (PONV) in orthopedic surgical patients," Journal of Holistic Nursing Science, vol. 8, no. 1, pp. 31-38, 2021.

[12] G. Xia, Y. Huang, F. Li et al., "A thermally flexible and multisite tactile sensor for remote 3D dynamic sensing imaging," Frontiers of Chemical Science and Engineering, vol. 14, no. 6, pp. 1039-1051, 2020.

[13] J. Y. Chen, B. S. Lin, Y. W. Luo, C. Y. Lin, and B. S. Lin, "Recovery evaluation system of bowel functions following orthopedic surgery and gastrointestinal endoscopy," IEEE Access, vol. 9, pp. 67829-67837, 2021.

[14] F. Y. Chang, P. T. Teng, and L. C. Chen, "Non-invasive monitoring method for lower-leg compartment syndrome using a wireless sensor system and finite element analysis," Proceedings of the Institution of Mechanical Engineers, Part H: Journal of Engineering in Medicine, vol. 235, no. 3, pp. 346-356, 2021.

[15] P. M. S. Shimabukuro, M. L. Duarte, A. M. Imoto et al., "Surgical Instruments Kits Orthopedic Electric Plaster Cutter Saw Price," Sao Paulo Medical Journal, vol. 138, no. 6, pp. 505$514,2020$.

[16] F. Gaetani, R. de Fazio, G. A. Zappatore, and P. Visconti, “A prosthetic limb managed by sensors-based electronic system: experimental results on amputees," Bulletin of Electrical Engineering and Informatics, vol. 9, no. 2, pp. 514-524, 2020.

[17] H. Amson, P. Lasselin, B. Naegels, G. P. Bracho, F. Aubrun, and M. Dziadzko, "Usability evaluation of sufentanil sublingual tablet analgesia in patients following enhanced recovery after surgery," Journal of Comparative Effectiveness Research, vol. 10, no. 9, pp. 743-750, 2021.

[18] T. A. Baldo, L. F. de Lima, L. F. Mendes, W. R. de Araujo, T. R. L. C. Paixão, and W. K. T. Coltro, "Wearable and biodegradable sensors for clinical and environmental applications," ACS Applied Electronic Materials, vol. 3, no. 1, pp. 68-100, 2020.

[19] H. Toda, M. Tada, T. Maruyama, and Y. Kurita, "Optimal swing support during walking using wireless pneumatic artificial muscle driver," Journal of Robotics and Mechatronics, vol. 33, no. 2, pp. 379-385, 2021.

[20] J. Meng, P. Pan, Z. Yang et al., "Degradable and highly sensitive CB-based pressure sensor with applications for speech recognition and human motion monitoring," Journal of Materials Science, vol. 55, no. 23, pp. 10084-10094, 2020.

[21] C. Weber, K. Fierz, M. Katapodi, and W. Hasemann, "An advanced practice nurse-led delirium consultation service reduces delirium severity and length of stay in orthopedic patients: a nonrandomized posttest only evaluation study," Perspectives in Psychiatric Care, vol. 56, no. 4, pp. 804-810, 2020.

[22] R. B. Green, M. Hays, M. Mangino, and E. Topsakal, “An anatomical model for the simulation and development of subcutaneous implantable wireless devices," IEEE Transactions on Antennas and Propagation, vol. 68, no. 10, pp. 7170-7178, 2020.

[23] M. Safaei, R. M. Meneghini, and S. R. Anton, "Compartmental force and contact location sensing in instrumented total knee replacements," Medical Engineering \& Physics, vol. 83, pp. 64-72, 2020.
[24] L. Witthauer, J. P. Cascales, E. Roussakis et al., "Portable oxygen-sensing device for the improved assessment of compartment syndrome and other hypoxia-related conditions," ACS Sensors, vol. 6, no. 1, pp. 43-53, 2020.

[25] L. Jiang and R. E. Mendame Ehya, "Effectiveness of a collaborative nursing care model for the treatment of patients with diabetic foot disease by transverse tibial bone transport technique: a pilot study," Journal of Peri Anesthesia Nursing, vol. 35 , no. 1 , pp. $60-66,2020$

[26] E. H. Othman, F. Shatnawi, O. Alrajabi, and J. A. Alshraideh, "Reporting nursing interventions classification and nursing outcomes classification in nursing research: a systematic review," International Journal of Nursing Knowledge, vol. 31, no. 1, pp. 19-36, 2020.

[27] K. Thompson, W. Griffiths-Jones, L. Frendin et al., "Interobserver agreement of sensor-derived compartmental pressure measurements in computer-assisted total knee arthroplasty," The Knee, vol. 27, no. 3, pp. 717-722, 2020.

[28] M. Pike, L. Campagna-Wilson, K. Sears, R. Warren, D. Legay, and D. Trudel, "Pilot study: the effectiveness of physiotherapyled screening for patients requiring an orthopedic intervention," Journal of Military, Veteran and Family Health, vol. 7, no. 2, pp. 3-15, 2021.

[29] H. Tilala, P. M. Suneesh, and K. J. J. Doss, “A study to assess the effectiveness of cryotherapy on level of pain among patient with arthralgia in selected orthopedic hospital at Rajkot," International Journal of Advances in Nursing Management, vol. 8, no. 1, pp. 69-71, 2020.

[30] S. Chae, H. Oh, and S. Moorhead, "Effectiveness of nursing interventions using standardized nursing terminologies: an integrative review," Western Journal of Nursing Research, vol. 42, no. 11, pp. 963-973, 2020. 